FERMILAB-Pub-03/165-T

LPM $/ 03-013$

UPRF-2003-12

\title{
An eigenvalue problem related to the non-linear $\sigma$-model: analytical and numerical results
}

\author{
V.A. Fateev ${ }^{*, \dagger}$ and E. Onofri ${ }^{\ddagger}, \S$
}

\begin{abstract}
An eigenvalue problem relevant for non-linear sigma model with singular metric is considered. We prove the existence of a non-degenerate pure point spectrum for all finite values of the size $R$ of the system. In the infrared (IR) regime (large $R$ ) the eigenvalues admit a power series expansion around IR critical point $R \rightarrow \infty$. We compute high order coefficients and prove that the series converges for all finite values of $R$. In the ultraviolet (UV) limit the spectrum condenses into a continuum spectrum with a set of residual bound states. The spectrum agrees nicely with the central charge computed by the Thermodynamic Bethe Ansatz method.
\end{abstract}

\footnotetext{
*Laboratoire de Physique Mathématique, Université Montpellier II, Pl. E. Bataillon, 34095 Montpellier, France, fateev@lpm.univ-montp2.fr

${ }^{\dagger}$ On leave of absence from Landau Institute for Theoretical Physics, ul.Kosygina 2, 117940 Moscow, Russia.

${ }^{\ddagger}$ Fermi National Accelerator Laboratory, P.O. Box 500, Batavia, Illinois, 60510, USA.

${ }^{\S}$ Permanent address: Dipartimento di Fisica, Università di Parma, I.N.F.N., Gruppo Collegato di Parma, 43100 Parma, Italy, onofri@unipr.it
} 


\section{INTRODUCTION}

The non-linear sigma models in two-dimensional (2D) space time are widely used in field theory as continuous models of two-dimensional spin systems (see e.g. Ref.s. 11, 2, 3, 4]) as well as in relation to string theory (e.g. Ref.s. [5, 6, 7, 8]). The general 2D sigma model $(\mathrm{SM})$ is defined through the action

$$
\mathcal{A}[G]=\frac{1}{2} \int G_{i j}(X) \partial_{\mu} X^{i} \partial_{\mu} X^{j} \mathrm{~d}^{2} x
$$

where the coordinates $x^{\mu}, \mu=1,2$ span a $2 \mathrm{D}$ flat space-time, while the fields $X^{i}, i=1, \ldots, d$ are coordinates in a $d$-dimensional Riemannian manifold called target space. The symmetric matrix $G_{i j}$ is the corresponding metric tensor.

The standard approach to 2D sigma models is perturbation theory. If the curvature of $G_{i j}$ is small, one can use the following one-loop renormalization group equation from Ref. [3]

$$
\frac{\mathrm{d}}{\mathrm{d} t} G_{i j}=-\frac{1}{2 \pi} R_{i j}
$$

where $t$ is the RG "time" (the logarithm of scale) and $R_{i j}$ is the Ricci tensor of $G$.

The simplest examples of $2 \mathrm{D}$ sigma models are SM with two-dimensional target space $(d=2)$. In this case $R_{i j}=\mathcal{R} \delta_{i j}$ where $\mathcal{R}$ is the scalar curvature. Then we can always choose (at least locally) conformal coordinates for which

$$
G_{i j}=e^{\Phi} \delta_{i j}
$$

with a single function $\Phi$.

An important role in the analysis of $2 \mathrm{D}$ sigma models is played by the effective central charge $c(R)$. This dimensionless function contains the information about the $\mathrm{UV}$ and $\mathrm{IR}$ properties of the theory and it is related to the ground state energy $E_{0}(R)$ of the corresponding quantum system, living on a finite space circle of length $R$ :

$$
E_{0}(R)=-\frac{\pi c(R)}{6 R}
$$

For SM with two-dimensional target space $c_{U V}=c(0)=2$.

In integrable theories this quantity can be calculated exactly using Thermodynamic Bethe Ansatz (TBA) equations Ref. 9, 10. This problem is however much more complicated for the excited levels $E_{i}(R)$, so it is useful to have some independent approach for their calculation. It was shown in Ref. 11] that for the sigma models with $d=2$, in one loop approximation (Eq12), this problem can be reduced to the eigenvalue problem for the operator:

$$
\widehat{h}=-\frac{1}{2} \nabla_{t}^{2}+\frac{1}{8} \mathcal{R}_{t}, \quad \widehat{h} \Psi_{i}=\frac{\pi e_{i}(R)}{6} \Psi_{i}
$$

Here $\nabla_{t}^{2}=e^{-\Phi}\left(\partial_{x}^{2}+\partial_{y}^{2}\right)$ is the Laplace operator and $\mathcal{R}_{t}$ is the scalar curvature in the SM metric renormalized at the scale $R$ :

$$
t-t_{0}=\log R \Lambda_{0}
$$

where $\Lambda_{0}$ is the normalization parameter. This operator is self-conjugate with respect to the scalar product in the SM metric:

$$
\left(\Psi_{1}, \Psi_{2}\right)=\int \Psi_{1}^{*} \Psi_{2} e^{\Phi} \mathrm{d} x \mathrm{~d} y
$$


The effective central charge $c(R)$ in one loop approximation can be expressed through the lowest eigenvalue :

$$
c(R)=2-e_{0}(R)
$$

and the excited levels $E_{i}(R)=E_{0}(R)+\pi\left(e_{i}(R)-e_{0}(R)\right) / 6 R$. We note that if in the IR limit SM flows to the critical point described by conformal field theory (CFT) then the numbers $\Delta_{i}=\left(e_{i}(\infty)-e_{0}(\infty)\right) / 24$ coincide with conformal dimensions of the fields in this CFT.

The eigenvalue problem (Eq. (5)) with the natural scalar product provided by the metric $G_{i j}(X)$ can be applied to the analysis of $2 \mathrm{D}$ sigma models with target space of arbitrary dimension $d$. It follows from Zamolodchikov's $c$-theorem that the effective central charge defined in Ref. 12] through the correlation functions of the energy momentum tensor is non-increasing as a function of the scale $R$. The effective central charge defined by Eq. (8) (with $d-e_{0}(R)$ in the r.h.s.) also satisfies this remarkable property, which follows from one of the results of Ref. [13], where it was shown that the lowest eigenvalue of the operator $\widehat{h}$ is a non-decreasing function of $R$.

A well known solution to Eq. (2) (see Ref. 11]) defines the axially symmetric metric of the "sausage" SM (an integrable deformation of the $O(3)$ non-linear sigma model), which is described by the action:

$$
\mathcal{A}_{s s g}=\int \frac{\left(\partial_{\mu} X\right)^{2}+\left(\partial_{\mu} Y\right)^{2}}{a(t)+b(t) \cosh 2 Y} \mathrm{~d}^{2} x
$$

where $a(t)=\nu \operatorname{coth} 2 u, b(t)=\nu / \sinh 2 u$, and $u=\nu\left(t_{0}-t\right) / 4 \pi$. It is easy to see from the explicit form of the metric (9) that operator $\widehat{h} / \nu$ depends only on the variable $u$ and does not depend on parameter $\nu$. It means that

$$
e_{i}^{s s g}(R)=\frac{\nu}{4 \pi} \kappa_{i}^{s s g}(u) .
$$

where after the substitution $\Psi=\exp (i m x) \Psi_{m}(y)(m \in \mathbb{Z})$ the scaling function $\kappa_{i}^{s s g}(u)$ is the eigenvalue of the Sturm-Liouville problem:

$$
\left[-\partial_{y}^{2}+m^{2}+\frac{1+\cosh 2 u \cosh 2 y}{(\cosh 2 u+\cosh 2 y)^{2}}-\frac{\frac{1}{6} \kappa_{i}^{s s g}(u) \sinh u}{\cosh 2 u+\cosh 2 y}\right] \Psi_{m}^{(i)}=0
$$

where the eigenfunctions $\Psi_{m}^{(i)}$ have finite norm according to Eq.(7). For the ground state $\kappa_{0}^{s s g}(u)$ this problem was studied in Ref. [11].

In this paper we consider the eigenvalue problem for the sigma model which correspond to another solution of RG Eq. (2). This solution can be obtained by analytic continuation $Y \rightarrow Y+i \pi / 4, u \rightarrow u+i \pi / 4$ from the solution for the sausage model. The corresponding action can be written as:

$$
\mathcal{A}=\int \frac{\left(\partial_{\mu} X\right)^{2}+\left(\partial_{\mu} Y\right)^{2}}{\alpha(t)+\beta(t) \sinh 2 Y} \mathrm{~d}^{2} x
$$

where $\alpha(t)=\nu \tanh 2 u, \beta(t)=\nu / \cosh 2 u$, and $u=\nu\left(t_{0}-t\right) / 4 \pi$. This metric has a singularity at $Y=-u$. It means that the coordinate $Y$ in target space should be considered only in the region $Y>-u$. The curvature $\mathcal{R}$ also has singularity at this point. However for small values of parameter $\nu$ the curvature is not small only in the narrow region $(\delta Y \sim \nu)$ in the vicinity of singularity. A more careful analysis shows that the one loop approximation is valid for the calculation of the observables in SM (12). The relative correction to one loop approximation as well as in the sausage SM has the order $\nu \log (1 / \nu)$. The eigenvalue 
equation for the scaling functions $\kappa_{i}(u)\left(e_{i}(R)=\nu \kappa_{i}(u) / 4 \pi\right)$ has now the form:

$$
\left[-\partial_{y}^{2}+m^{2}-\frac{1-\sinh 2 u \sinh 2 y}{(\sinh 2 u+\sinh 2 y)^{2}}-\frac{\frac{1}{6} \kappa_{i}(u) \cosh u}{\sinh 2 u+\sinh 2 y}\right] \Psi_{m}^{(i)}=0
$$

The solution $\Psi_{m}^{(i)}(y)$ should satisfy now the boundary condition

$$
\Psi_{m}^{(i)}(y) \underset{y \rightarrow-u}{\approx}(y+u)^{1 / 2}
$$

and it must be square integrable with respect to the natural norm

$$
\left\|\Psi_{m}^{(i)}\right\|^{2}=\int_{-u}^{\infty} \frac{\left|\Psi_{m}^{(i)}(y)\right|^{2}}{\sinh 2 u+\sinh 2 y} \mathrm{~d} y
$$

In the IR limit $u \rightarrow-\infty$ the metric (12) has an asymptotic which can be written in the form of Eq. (3) with $\exp \left\{-\Phi_{I R}\right\}=\frac{1}{2} \nu(\exp \{2 Z\}-1)$, with $Z=Y+u$. For discrete values of the parameter $\nu=4 \pi / N$ the SM with this metric can be derived from the $S U(2)$ level $N$ WZW models by gauging $U(1)$ symmetry (see Ref. [14, 15] for details). The resulting $S U(2)_{N} / U(1)$ coset model described by the SM with metric $\exp \left(\Phi_{I R}\right) \delta_{i j}$ coincides with $Z_{N}$ parafermionic CFT of Ref. 16. For general values of $u$ the quantum field theory (QFT) corresponding to SM (12) can be considered as the deformation of parafermionic CFT. It is natural to expect that it will be a massless theory describing the RG flow from rather nontrivial UV field theory (which is also well defined for the same discrete values of parameter $\nu)$ with $c_{U V}=2$ to the parafermionic CFT with $c_{I R}=2-6 /(N+2)$ in the IR limit. The scaling functions $\kappa_{i}(u)$ in this case describe the RG dynamics of energy levels from UV regime to IR asymptotics, where they define (with relative accuracy $O(1 / N)$ ) the spectrum of anomalous dimensions of parafermionic CFT (see Appendix C).

This massless QFT is integrable and can be described in terms of factorized scattering theory for massless excitations. For $\nu=4 \pi / N$ with $N \geq 3$ the ground state energy Eq. (4) of the SM (12) (as well as that of the sausage SM (9), of Ref.11]) admits an exact calculation by TBA method. These equations will be described in Sec. 5 .

Both eigenvalue problems Eq. $(11,13)$ are believed to have a purely discrete spectrum. The ground state eigenvalue $\kappa_{0}^{s s g}$ of Eq. (111) was studied in Ref. 11], where the asymptotics of this function in the regimes $u \rightarrow 0$ and $u \rightarrow \infty$ were found. This function was studied numerically in Ref. [17] and the result was in perfect agreement with the scaling function calculated from TBA equations (see Sec.5). Actually it is rather easy today to attack the spectral problem Eq. (11) by using sparse matrix techniques available in mathematical libraries. Good accuracy can be achieved by introducing a multi-grid discretization and using Richardson extrapolation. This approach, however, is not immediately applicable to new equation (13) due to its singular nature. In the following we shall bring the equation to a form which is suitable for a detailed perturbative analysis (Sec. 2), to a second form which allows an accurate asymptotic analysis in the UV regime (Lamé form, Sec. 31), as well to a third form, more suitable for a purely numerical approach (Sec. 4). Finally (Sec. (5) we shall exhibit the matching of the ground state of Eq. (13) with the central charge of the modified TBA system whose structure is given in Fig. 8. The interested reader will find some further mathematical details in the Appendices. 


\section{The Connection with Heun's equation}

To bring Eq. (13) to a more manageable form, we begin by re-absorbing the the integration measure into the wave function. By defining

$$
\begin{aligned}
\Psi(y) & =\sqrt{\rho(y)} \phi(y), \\
\rho(y) & =\sinh 2 y+\sinh 2 u
\end{aligned}
$$

we find

$$
-\frac{\mathrm{d}}{\mathrm{d} y}\left(\rho(y) \frac{\mathrm{d} \phi(y)}{\mathrm{d} y}\right)+\left(m^{2} \rho(y)-\sinh 2 y\right) \phi(y)=\frac{1}{6} \kappa \cosh 2 u \phi(y)
$$

Putting $x=e^{-2(y+u)}$ and $w=-e^{-4 u}$, Eq. (17) is transformed into the following

$$
\phi^{\prime \prime}(x)+\left(\frac{1}{x-1}+\frac{1}{x-w}\right) \phi^{\prime}(x)+\frac{\left(1-m^{2}\right) x^{2}-\frac{1}{6} \kappa(1-w) x+m^{2}+w}{4 x^{2}(x-1)(x-w)} \phi(x)=0
$$

of the Fuchsian type. A further substitution $\phi \rightarrow \sqrt{ } x f(x)$ reduces Eq. (18) to the form

$$
f^{\prime \prime}(x)+\left(\frac{m+1}{x}+\frac{1}{x-1}+\frac{1}{x-w}\right) f^{\prime}(x)+\frac{(1+m) x-\mathfrak{q}}{x(x-1)(x-w)} f(x)=0
$$

where the so-called accessory parameter $\mathfrak{q}$ is given by

$$
\mathfrak{q}=\frac{1}{24}(\kappa(1-w)+6(1+w)(1+2 m)) .
$$

This equation was analyzed by Heun in 1888 (see Ref. [18, 19]) who considered a general linear differential equation of the second order with four Fuchsian singularities. In Heun's notation, the solution is formally given by $F(w ; \mathfrak{q} ; 1+m, 1,1+m, 1 ; x)$, but this is of little use in practice. We gain some insight from the fact that the limit $w \rightarrow-\infty$ is a case of confluence of singularities which takes us back to the hypergeometric equation (see Ref. [19] for a general treatment).

2.1. The eigenvalue problem in algebraic form. It is well known that series solutions for Heun's equations can be most conveniently constructed using the basis of Jacobi polynomials $P_{n}^{(m, 0)}(1-2 x)$ (see e.g. 20], Vol.III). We are now going to show how to solve the eigenvalue problem by exploiting this favorable basis: the problem will reduce to finding the spectrum of an (infinite-dimensional) tridiagonal matrix for which efficient algorithms are well-known to exist [21]. Let us consider Eq. (19): after setting $y=1-2 x$, we can easily expand the solution in a series of Jacobi polynomials $\mathcal{P}_{n}^{m} \equiv P_{n}^{(m, 0)}(y)$ by converting the differential equation in the form

$$
\mathcal{H} f \equiv\left\{(1-2 w-y) \widehat{N}(\widehat{N}+m+1)+\left(1-y^{2}\right) \frac{\mathrm{d}}{\mathrm{d} y}-(1+m) y\right\} f=(2 \mathfrak{q}-m-1) f
$$

where $\widehat{N} \mathcal{P}_{n}^{m}=n \mathcal{P}_{n}^{m}$. Now we can use the basic properties of Jacobi's polynomials (see e.g. [20], Vol.II, [22] ) to reduce the operator $\mathcal{H}$ to the form $\mathcal{H} f=(1-2 w) \mathcal{H}_{0} f+\mathcal{V} f$ whose action on the basis vectors is particularly simple:

$$
\begin{aligned}
\mathcal{H}_{0} \mathcal{P}_{n}^{m} & =n(n+m+1) \mathcal{P}_{n}^{m} \\
\mathcal{V} \mathcal{P}_{n}^{m} & =m \frac{(2+m) n^{2}+(m+1)(m+2) n+m(m+1)}{(m+2 n)(m+2 n+2)} \mathcal{P}_{n}^{m} \\
& -2 \frac{n^{2}(m+n)^{2}}{(m+2 n)(m+2 n+1)} \mathcal{P}_{n-1}^{m}-2 \frac{(n+1)^{2}(m+n+1)^{2}}{(m+2 n+1)(m+2 n+2)} \mathcal{P}_{n+1}^{m}
\end{aligned}
$$

We can now conveniently study the spectrum of $\mathfrak{q}$ using this tridiagonal matrix representation, by applying, for instance, the technique of Sturm sequences and bisection [21]. The 
matrix representation also lends itself to a very simple perturbation series expansion, as we discuss in the next section. We shall have to refer to the matrix representation of $\mathcal{H}$ in the orthonormal basis $\phi_{n}^{m}=(-)^{n} \sqrt{\frac{1}{2 n+m+1}} \mathcal{P}_{n}^{m}$ as $V_{n n^{\prime}}$ :

$$
\begin{aligned}
V_{n+1, n} & =\frac{2(n+1)^{2}(n+m+1)^{2}}{(2 n+m+2) \sqrt{(2 n+m+2)^{2}-1}} \\
V_{n-1, n} & =\frac{2 n^{2}(n+m)^{2}}{(2 n+m) \sqrt{(2 n+m)^{2}-1}}
\end{aligned}
$$

diagonal terms being unchanged, but the former $\mathcal{V}$, being rational in its indices, is more convenient for the calculation of perturbative coefficients; we show in Appendix A, that we can use $\mathcal{V}$ without modifying the standard algorithm.

2.2. Perturbation theory. The tridiagonal matrix representation of $\mathcal{H}$, when rewritten as $\mathcal{H}=\varepsilon^{-1}\left(\mathcal{H}_{0}+\varepsilon \mathcal{V}\right)$, can be used to calculate a perturbative expansion in the parameter

$$
\varepsilon=(1-2 w)^{-1}=\left(1+2 e^{-4 u}\right)^{-1} .
$$

The convergence of the expansion is governed by the Kato-Rellich theorem ${ }^{\text {: }}$ let there exist constants $a, b$ such that $\|\mathcal{V} \phi\| \leq a\|\phi\|+b\left\|\mathcal{H}_{0} \phi\right\|$ (\|.\| denotes $L_{1}$-norm). Then the perturbative expansion defines a regular analytic function for $|b \varepsilon|<1$. For a tridiagonal matrix it's not so difficult to find norm estimates; in our case it's simple algebra to check that the column sums of the matrix elements of $\mathcal{V}$ coincide with the diagonal matrix elements of $\mathcal{H}_{0}$, up to an additive constant, therefore we have

$$
\mathcal{V}=\mathcal{S}\left(\mathcal{H}_{0}+(m+1) \mathbb{I}\right)
$$

with $\mathcal{S}$ a stochastic matrix $\|$ and $\mathbb{I}$ the identity matrix. Hence it follows

$$
\|\mathcal{V} \phi\|=\left\|\mathcal{S}\left(\mathcal{H}_{0}+(m+1) \mathbb{I}\right) \phi\right\| \leq\left\|\mathcal{H}_{0} \phi\right\|+(m+1)\|\phi\|
$$

which implies that the perturbative series will converge for $\varepsilon<1$, that is for all $u$. Actually we can say more: let us denote by $R(H, \mu)=(H+\mu)^{-1}$ the resolvent operator; by applying Schur's Test to the matrix $R\left(\mathcal{H}_{0}, \mu\right) V$ (the symmetric version of $\mathcal{V}$ ) one concludes that

$$
\left\|R\left(\mathcal{H}_{0}, \mu\right) V\right\| \leq 1
$$

if $\mu \geq m+13 / 8$. Now the resolvent of $\mathcal{H}$ satisfies Lippman-Schwinger equation

$$
R(\mathcal{H}, \mu)=R\left(\mathcal{H}_{0}, \mu\right)-\varepsilon R\left(\mathcal{H}_{0}, \mu\right) V R(\mathcal{H}, \mu)
$$

which for $|\varepsilon|<1$ can be inverted to give

$$
R(\mathcal{H}, \mu)=\left(1+\varepsilon R\left(\mathcal{H}_{0}, \mu\right) V\right)^{-1} R\left(\mathcal{H}_{0}, \mu\right) .
$$

Since $R\left(\mathcal{H}_{0}, \mu\right)$ is a compact operator, Eq. (29) is telling us that the resolvent $R(\mathcal{H}, \mu)$ is itself a compact operator, being the product of a compact operator with a bounded one. This implies that the spectrum of $\mathcal{H}$ is purely discrete.

The expansion can now be computed rather easily by the standard recursive algorithm (see Appendix A). Details on the series expansion can be found in Appendix B, where we prefer to adopt a different parameter which naturally appears in the Lamé formulation of next section, namely

$$
\lambda=\left(1+e^{-4 u}\right)^{-1}=\frac{2 \varepsilon}{1+\varepsilon}, \varepsilon=\frac{\lambda}{2-\lambda} .
$$

\footnotetext{
"See for instance 24] 25]

${ }_{i \text {.e. }} \sum_{i} \mathcal{S}_{i, j} \equiv 1$
} 
The expansion in powers of $\lambda$ turns out to be simpler and with better convergence properties; indeed the substitution $\varepsilon \rightarrow \lambda$ is just a special case of Euler's $(E, q)$-method [26]. We present just a sample of the infinite number of different series expansions, since we believe that nobody would like to copy them from paper but would rather prefer getting the code which generated the expansion**. The first few terms for the ground state value of $\kappa$ at fixed $m$ are the following

$$
\begin{aligned}
\frac{1}{6} \kappa_{m, 0}= & 1+2 m-\frac{2 m}{m+2} \lambda-\frac{4(m+1)^{3}}{(m+2)^{3}(m+3)} \lambda^{2}- \\
& -\frac{8(m+1)^{3}\left(2 m^{2}+5 m+4\right)}{(m+2)^{5}(m+3)(m+4)} \lambda^{3}+O\left(\lambda^{4}\right)
\end{aligned}
$$

while for the excited states, after putting $j=\frac{1}{2} m+n$, we have (see also Appendix C)

$$
\begin{aligned}
\frac{1}{6} \kappa_{m, n}= & (2 j+1)^{2}-m^{2}-\frac{\left[4 j(j+1)-m^{2}\right]^{2}}{8 j(j+1)} \lambda- \\
& -\frac{1}{2^{9}(2 j+1)}\left[\frac{\left(4(j+1)^{2}-m^{2}\right)^{4}}{(j+1)^{3}(2 j+3)}-\frac{\left(4 j^{2}-m^{2}\right)^{4}}{j^{3}(2 j-1)}\right] \lambda^{2}+O\left(\lambda^{3}\right) .
\end{aligned}
$$

Using floating point arithmetic we may quickly explore very high orders, with due attention to truncation errors which accumulate along the iteration. The asymptotic behavior of the coefficients shows very clearly a limit $c_{n+1} / c_{n} \rightarrow 1$, confirming that the series converges in the unit circle, which means in the domain $\left|e^{4 u} /\left(1+e^{4 u}\right)\right|<1$. In the complex $u$ plane this is a domain which includes the whole real axis. However as we venture along the positive real axis, the convergence is critically slowed down: to go deep in the UV region we may be obliged to sum a really huge number of terms, or try some resummation, e.g. via Padé approximants. Since high order coefficients are easily computed, however, we may try to extract the asymptotic behavior of $\kappa(u)$ for large positive $u$ by analyzing the asymptotic behavior of the coefficients. For example we can verify that the UV asymptotics of the ground state eigenvalue $\kappa_{0}(u)$ coincides with that of function $\kappa_{0}^{s s g}(u)$ (see Sec. 3) and has a form:

$$
\kappa_{0}(u)=\frac{3 \pi^{2}}{2(u+\log 4)^{2}}+O\left(1 / u^{5}\right)
$$

When expressed in terms of $\varepsilon$ this formula can be expanded in a power series and the coefficients compared to those coming from perturbation theory. Sub-dominant terms tend to mask the simple $n^{-1}(\log n)^{-3}$ behavior one should expect; we find that it is more accurate to compare the Taylor coefficients of the function $1 /(4 \mathfrak{q}-1)$, which is actually diverging at $\varepsilon \rightarrow 1$, with those of its leading term $O\left(\log (1-\varepsilon)^{2}\right)$. However, to make the comparison even more transparent, we may look for a special function whose behavior at $\varepsilon \rightarrow 1$ is the simplest possible. Let us observe that $2 \mathfrak{q}-1$ turns out to be an odd function of $\varepsilon$; the new function $\sqrt{\varepsilon /(4 \mathfrak{q}-2+\varepsilon)}$ has the simple $O(\log (1-\varepsilon))$ leading singular behavior at $\varepsilon \rightarrow 1$, and it is an even function of $\varepsilon$. We argue that its leading behavior should then be

$$
\Upsilon(\varepsilon) \approx \frac{1}{2 \pi \varepsilon} \log \left(\frac{1+\varepsilon}{1-\varepsilon}\right) \text {. }
$$

In fact we find that the expansion of $\Upsilon$ matches the perturbative series with a high accuracy (see Fig. 1 where the deviation is magnified $10^{5}$ times). The asymptotic behavior will be recovered in a very precise way numerically in Sec. 4 hence its extraction from the

** Matlab and Mathematica codes are available at the web site www.fis.unipr.it/ onofri. 


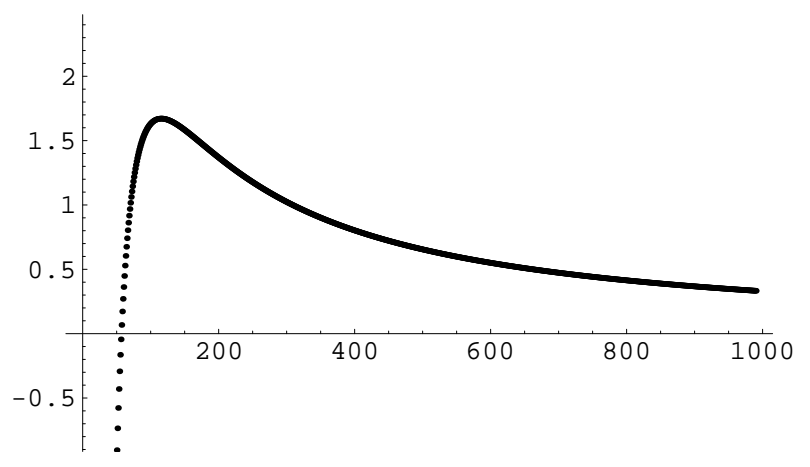

FiguRE 1. Relative deviation of $\Upsilon$ 's expansion coefficients from those of its leading asymptotics $\left(\times 10^{5}\right)(\mathrm{Eq}$ 341) .

perturbative series appears of purely academic interest. Anyhow, assuming Eq. (34) it follows from Eq. (20)

$$
\kappa \equiv \frac{24 \mathfrak{q}-6(1+w)}{1-w} \approx 6(4 \mathfrak{q}-1)=6 / \Upsilon^{2} \approx \frac{3 \pi^{2}}{2 u^{2}}
$$

since $\frac{1}{4} \log ((1+\varepsilon) /(1-\varepsilon))=\frac{1}{4} \log \left(1+e^{4 u}\right) \approx u$ as $\varepsilon \rightarrow 1$.

2.3. Sausage model equation. The sausage model was our starting point. Now we go back to it and show how it fits into the correspondence with Heun's equation. The scaling function is defined in Eq. (111). We shall now look for its algebraic equivalent as we did in Sec. 2. Since the two equations are related by analytic continuation, it will not come out as a surprise that the differential equation is the same, up to a map $w \rightarrow \bar{w}, \mathfrak{q} \rightarrow \overline{\mathfrak{q}}$. The range of values for the problem of SM(12) is $\frac{1}{4}<\mathfrak{q}<\frac{1}{2}, w<0$, while the sausage is characterized by $\frac{1}{2}<\overline{\mathfrak{q}}<1, w>0$. The point is that Eq. (11) can be brought to Heun's form by the transformation $\xi=\exp \{2(y+u)\}$. The singularities are now located at $\{0,-1,-w, \infty\}$, with $w=\exp \{4 u\}$ and $24 \mathfrak{q}=6(1+w)+\kappa(u)(w-1)$. The equation is actually the same as the one we find for the SM(12), but the domain involved is the positive real line instead of the unit interval and the singularities are differently situated. By applying well-known transformation properties of Heun's equation (Ref. [18, 23]) we can reposition the domain on the unit interval $(0,1)$, the singular points being now $0,1, \bar{w}=w /(w-1), \infty$ and the new accessory parameter is given by

$$
\overline{\mathfrak{q}}=\frac{w-\mathfrak{q}}{w-1} .
$$

Notice that the map $\{\mathfrak{q}, w\} \rightarrow\{\overline{\mathfrak{q}}, \bar{w}\}$ is involutory with $w=\infty$ as the only fixed point, the interval $\frac{1}{2}<\overline{\mathfrak{q}}<1$ being mapped onto $1<\mathfrak{q}<\infty$.

Hence we can use the same algorithm of the previous section in a different domain. The "magic" here is provided by the analyticity properties of the models involved. The complex shift transforming the "sausage" model into the SM (12) does not modify very much the eigenvalue equation, which turns out to be the same equation in a different domain.

\section{The LAmÉ FORMulation}

The parameter $\lambda$ defined by Eq.(30) is naturally related with a reformulation of Eq. (13) close to Lamé elliptic equation. If we define the modulus of Jacobi elliptic functions

$$
k^{2}=\lambda=1 /(1+\exp (-4 u))
$$


then the substitution

$$
e^{y-u}=\frac{\operatorname{dn}\left(z \mid k^{2}\right)}{k \operatorname{sn}\left(z \mid k^{2}\right)}, \quad \psi_{m}=\sqrt{\frac{\operatorname{sn}\left(z \mid k^{2}\right) \operatorname{dn}\left(z \mid k^{2}\right)}{\operatorname{cn}\left(z \mid k^{2}\right)}} \Psi_{m}
$$

maps the point $y=\infty$ to $z=0$, the point $y=-u$ to $z=K$, where $K\left(k^{2}\right)$ is the real period of Jacobi elliptic functions, and it turns Eq. (13) to the form:

$$
\left(-\frac{\mathrm{d}^{2}}{\mathrm{~d} z^{2}}-\frac{\mathrm{dn}^{2}\left(2 z \mid k^{2}\right)}{\mathrm{sn}^{2}\left(2 z \mid k^{2}\right)}+\frac{m^{2} \mathrm{cn}^{2}\left(z \mid k^{2}\right)}{\operatorname{sn}^{2}\left(z \mid k^{2}\right) \mathrm{dn}^{2}\left(z \mid k^{2}\right)}\right) \psi_{m, n}=\frac{1}{6} \kappa_{m, n} \psi_{m, n}
$$

with the boundary conditions $\psi_{m} \sim z^{m+1 / 2}$ at $z \rightarrow 0 ; \psi_{m} \sim(K-z)^{1 / 2}$ at $z \rightarrow K$. This equation can be studied analytically in two limits $k^{2} \rightarrow 0(u \rightarrow-\infty)$ and $k^{2} \rightarrow 1(u \rightarrow-\infty)$. In the first case we can develop the standard perturbation theory near the exact solutions $\psi_{m, n}(z)=\sqrt{\sin 2 z} \cos ^{m}(z) P_{n}^{(0, m)}(\cos 2 z)$ where $P_{n}^{(\alpha, \beta)}(x)$ are Jacobi polynomials. This perturbation theory gives the same IR expansion for the eigenvalues which was considered in previous section.

In the opposite limit $u \rightarrow \infty, k \rightarrow 1$ and the real period $K \sim-\frac{1}{2} \log \left(\left(1-k^{2}\right) / 16\right) \sim$ $2 u+2 \log 2 \rightarrow \infty$. In this case the potential term in Eq.(39) is equal to $m^{2}$ almost everywhere and near the points $z=0 ; z=K$ it can be approximated with exponential in $u$ accuracy by the potentials:

$$
V(z)=-\frac{1}{\sinh ^{2} 2 z}+m^{2} \operatorname{coth}^{2} z \quad 0<z \ll K
$$

and

$$
V_{1}\left(z_{1}\right)=-\frac{1}{\sinh ^{2} 2 z_{1}}+m^{2} \tanh ^{2} z_{1} \quad 0<z_{1} \equiv K-z \ll K
$$

Both these potentials appeared in Ref. [27], where the spectrum of CFT describing Witten's two-dimensional Euclidean black hole [28] was studied. There, it was noted that potential $V_{1}\left(z_{1}\right)$ is attractive and has the bound states solutions:

$$
\psi_{m, n}=\sqrt{\tanh z_{1}}\left(\cosh z_{1}\right)^{2 n-m+1} F\left(-n,-n+m, m-2 n ; 1-\tanh ^{2} z_{1}\right)
$$

where $F(a, b, c, z)$ is Gauss' hypergeometric function. These solutions are normalizable for integer $n<(m-1) / 2$ and give the levels

$$
\frac{1}{6} \kappa=\left\{m^{2}-(2 n+1-m)^{2} \mid n=0,1, \ldots,\left[\frac{1}{2} m\right]-1\right\} .
$$

The corresponding eigenvalues of Eq.(39) approach these levels exponentially in $u$.

The potential $V(z)$ is repulsive and does not have normalizable solutions. For $2 n \geq m-1$ we parameterize $\kappa_{m, n} / 6=m^{2}+p_{n}^{2}$. Then solutions regular at $z=0$ and at $z_{1}=K-z=0$ are found to be

$$
\begin{gathered}
\psi_{m, n}(z)=(\tanh z)^{m+\frac{1}{2}}(\cosh z)^{i p} F\left(\frac{1}{2}(1+m-i p), \frac{1}{2}(1+m-i p), m+1 ; \tanh ^{2} z\right) \\
\psi_{m, n}\left(z_{1}\right)=\left(\tanh z_{1}\right)^{\frac{1}{2}}\left(\cosh z_{1}\right)^{i p} F\left(\frac{1}{2}(1+m-i p), \frac{1}{2}(1-m-i p), 1 ; \tanh ^{2} z_{1}\right)
\end{gathered}
$$

Matching these solutions with the plane wave solution in the region $0 \ll z \ll K$ we obtain the quantization condition: $p_{n}=\frac{1}{4} \pi(2 n-m+2) /\left(u+r_{m}\right)$ where $r_{m}=\psi(1)-\psi\left(\frac{1}{2} m+\frac{1}{2}\right)$, and $\psi(x)$ is the logarithmic derivative of the $\Gamma$ function. This condition leads to the asymptotics

$$
\frac{1}{6} \kappa_{m, n}(u)=m^{2}+\pi^{2} \frac{(2 n-m+2)^{2}}{16\left(u+r_{m}\right)^{2}}+O\left(1 / u^{5}\right) ; \quad n \geq \frac{1}{2}(m-1)
$$


(see Fig. (4). We note that for $m \neq 0$ this UV behavior is different from that for the sausage model eigenvalues, which is given by

$$
\frac{1}{6} \kappa_{m, n}^{s s g}(u)=m^{2}+\pi^{2} \frac{(n+1)^{2}}{4\left(u+r_{m}\right)^{2}}+O\left(1 / u^{5}\right) ; \quad(n \geq 0)
$$

The sausage model eigenvalue problem of Eq. (11) can also be written in the elliptic form of Ref. [1] with $k_{s}^{2}=1-\exp (-4 u)$ :

$$
\left(-\frac{\mathrm{d}^{2}}{\mathrm{~d} z^{2}}-\frac{\mathrm{cn}^{2}\left(2 z \mid k_{s}^{2}\right)}{\mathrm{sn}^{2}\left(2 z \mid k_{s}^{2}\right)}+\frac{m^{2} \mathrm{dn}^{2}\left(z \mid k_{s}^{2}\right)}{\operatorname{sn}^{2}\left(z \mid k_{s}^{2}\right) \mathrm{cn}^{2}\left(z \mid k_{s}^{2}\right)}\right) \psi_{m, n}=\frac{\kappa_{m, n}^{s s g} k_{s}^{2}}{6} \psi_{m, n}
$$

with boundary conditions $\psi_{m} \sim z^{m+1 / 2}$ at $z \rightarrow 0 ; \psi_{m} \sim(K-z)^{m+1 / 2}$ at $z \rightarrow K$. In the UV limit $u \rightarrow \infty$ the potential term in this equation at the both ends tends to $V$ given by Eq.(40). The normalizable solutions do not appear and both asymptotics can be described by the eigenfunction (44). The quantization condition for parameter $p_{n}$ in this case leads to the asymptotics Eq.(47).

\section{NUMERICAL ANALYSIS}

The matrix representation introduced in a previous section, while useful from the analytic viewpoint, is not the best choice if we want to compute the spectrum beyond perturbation theory. Actually an $n$-dimensional truncation of the matrix given in Eq. (22) is going to be essentially equivalent to $n$-th order perturbation theory. We are now introducing another transformation of Eq. (13) which will allow us to efficiently explore the whole range $-\infty<u<\infty$.

We start from the fact that in the limit $u \rightarrow-\infty$ (and $m=0$ ) there is a simple solution with

$$
\Psi(y)=\sqrt{1-e^{-2(y+u)}} \equiv \sigma(y),
$$

with $\kappa(u) \rightarrow 6$. Hence it seems promising to look for a solution of the form

$$
\Psi(y)=\sigma(y) \psi(y)
$$

To find the new differential equation it is convenient to write down the functional $\langle\mathcal{H}\rangle$ whose critical points are the eigenvalues: by denoting $\chi(y) \equiv 1-\sinh 2 u \sinh 2 y$ and recalling $\rho(y)$ from Eq. (16), we have

$$
\langle\mathcal{H}\rangle=\frac{\left.\int_{-u}^{\infty}\left(\Psi\left(m^{2}-\partial^{2}\right) \Psi-\chi \rho^{-2}\right) \Psi^{2}\right) \mathrm{d} y}{\int_{-u}^{\infty} \rho^{-1} \Psi^{2} \mathrm{~d} y}
$$

Now, by inserting Eq. (50), after an integration by part, we find

$$
\langle\mathcal{H}\rangle=\frac{\int_{-u}^{\infty}\left[\sigma^{2} \psi^{\prime 2}+\left(\sigma^{\prime 2}-\chi \sigma^{2} / \rho^{2}-\left(\sigma \sigma^{\prime}\right)^{\prime}+m^{2} \sigma^{2}\right) \psi^{2}\right] \mathrm{d} y}{\int_{-u}^{\infty} \sigma^{2} \rho^{-1} \psi^{2} \mathrm{~d} y}
$$

The variational equation resulting from this functional

$$
-\frac{\mathrm{d}}{\mathrm{d} y}\left(\sigma^{2} \frac{\mathrm{d} \psi}{\mathrm{d} y}\right)+V \psi=\frac{1}{6} \kappa \cosh 2 u \sigma^{2} \rho^{-1} \psi
$$

suggests to introduce a new coordinate $\xi$ such that $\mathrm{d} y=\sigma^{2} \mathrm{~d} \xi$; we find

$$
y=-u+\frac{1}{2} \log \left(1+e^{2 \xi}\right)
$$

and the equation simplifies to

$$
-\frac{\mathrm{d}^{2} \psi}{\mathrm{d} \xi^{2}}+\sigma^{2} V(\xi, u) \psi=\frac{1}{6} \kappa(u) W(\xi, u) \psi(\xi)
$$


with

$$
\begin{aligned}
\sigma^{2} V & =\frac{e^{2 \xi}\left(\left(1+e^{4 u}\right)^{2}+\left(1+e^{2 \xi}\right)^{2}-1+e^{4 u}\left(e^{4 \xi}+3 e^{2 \xi}\right)\right)}{\left(1+e^{2 \xi}\right)^{2}\left(1+e^{4 u}+e^{2 \xi}\right)^{2}}+\frac{1}{4} m^{2}(1+\tanh \xi)^{2} \\
W & =\frac{e^{2 \xi}\left(1+e^{4 u}\right)}{\left(1+e^{2 \xi}\right)\left(1+e^{4 u}+e^{2 \xi}\right)}
\end{aligned}
$$

The crucial property of this new formulation is that it is now regular on the whole real axis. With appropriate boundary conditions (Neumann's b.c. $)^{\dagger \dagger}$ the eigenvalue equation can now be easily solved by standard sparse-matrix generalized eigenvalue routines ${ }^{\ddagger}$
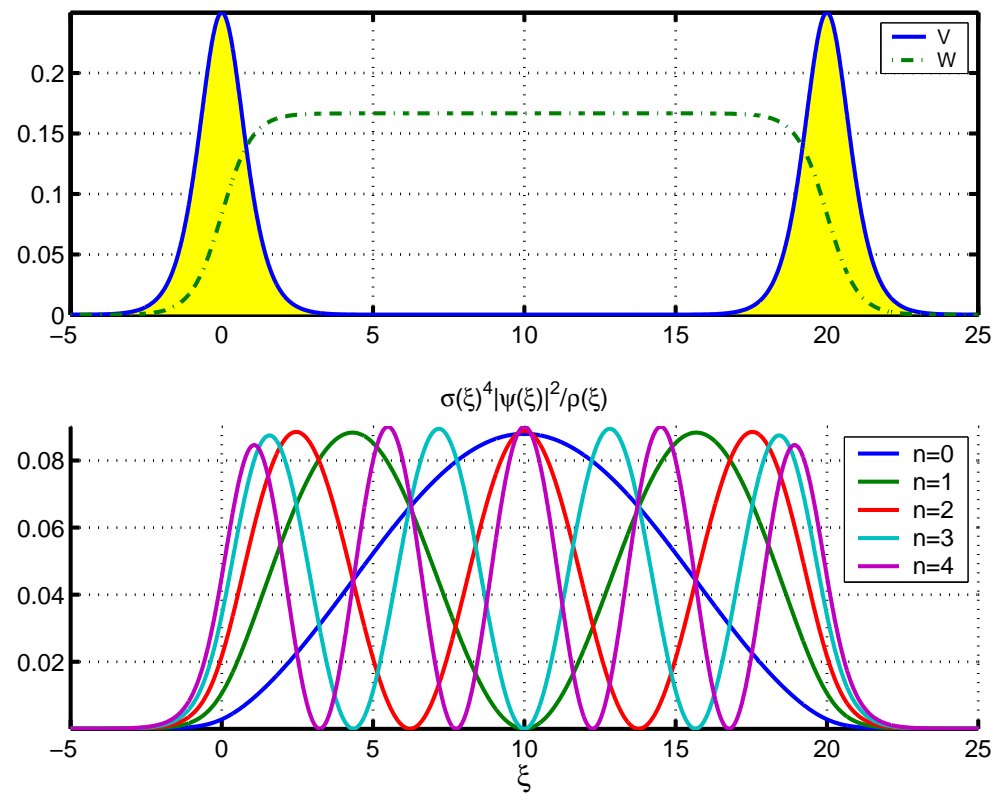

FiguRE 2. The generalized potentials $V, W$ (upper) and the first eigenfunctions $\psi_{n}^{2}$ for $u=10, m=0$.

See Fig.s (23) for some typical waveforms. The density includes the measure appropriate for the new variable $\xi$, namely $\sigma(\xi)^{4} / \rho(\xi)$. In the limit $u \rightarrow-\infty$ we easily recover the discrete spectrum

$$
\frac{1}{6} \kappa=\left\{(2 j+1)^{2}-m^{2} \mid j=n+\frac{1}{2} m, n=0,1,2, \ldots\right\}
$$

as we already know from perturbation theory (see Eq. (33)). In the other limit, $u \gg 0$, the spectrum can be described as a monotonously decreasing flow toward $\kappa / 6=m^{2}$, except for a finite number of eigenvalues which have a value less than $m^{2}$ : these peculiar "bound states" are given by Eq. (43) and they are easily reproduced numerically (see Fig. (4). We easily check that they nicely agree with the asymptotic formulas already given in Eq. (47).

\section{MATChing TO TBA DATA}

As it was already mentioned in Introduction for the special values of parameter

$$
\frac{\nu}{4 \pi}=\frac{1}{N} ; \quad N=3,4, \ldots
$$

\footnotetext{
${ }^{\dagger \dagger}$ It is somewhat tedious to trace the b.c. back from the original equation; suffices to say that, intuitively speaking, since the constant solution is exact in the limit $u \rightarrow-\infty$, Neumann b.c. are the natural ones.

${ }^{\ddagger}$ We successfully used the routine eigs in Matlab.
} 
a)
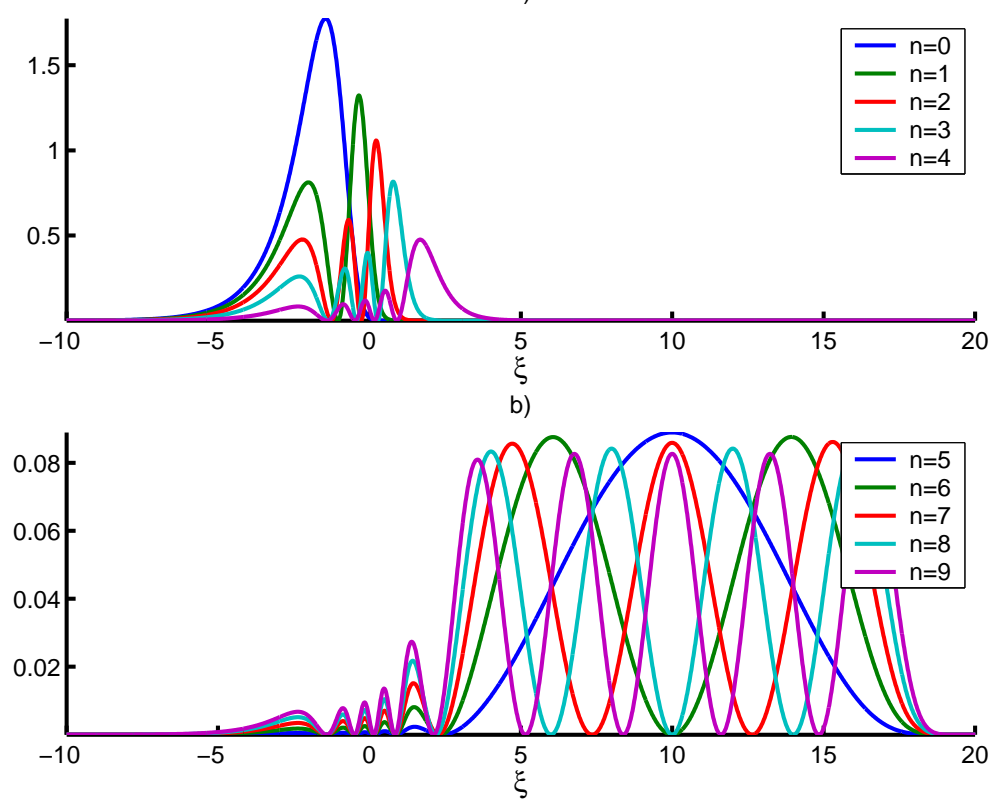

Figure 3. The densities at $u=10, m=10$ : a) "bound states", b) the "continuum".

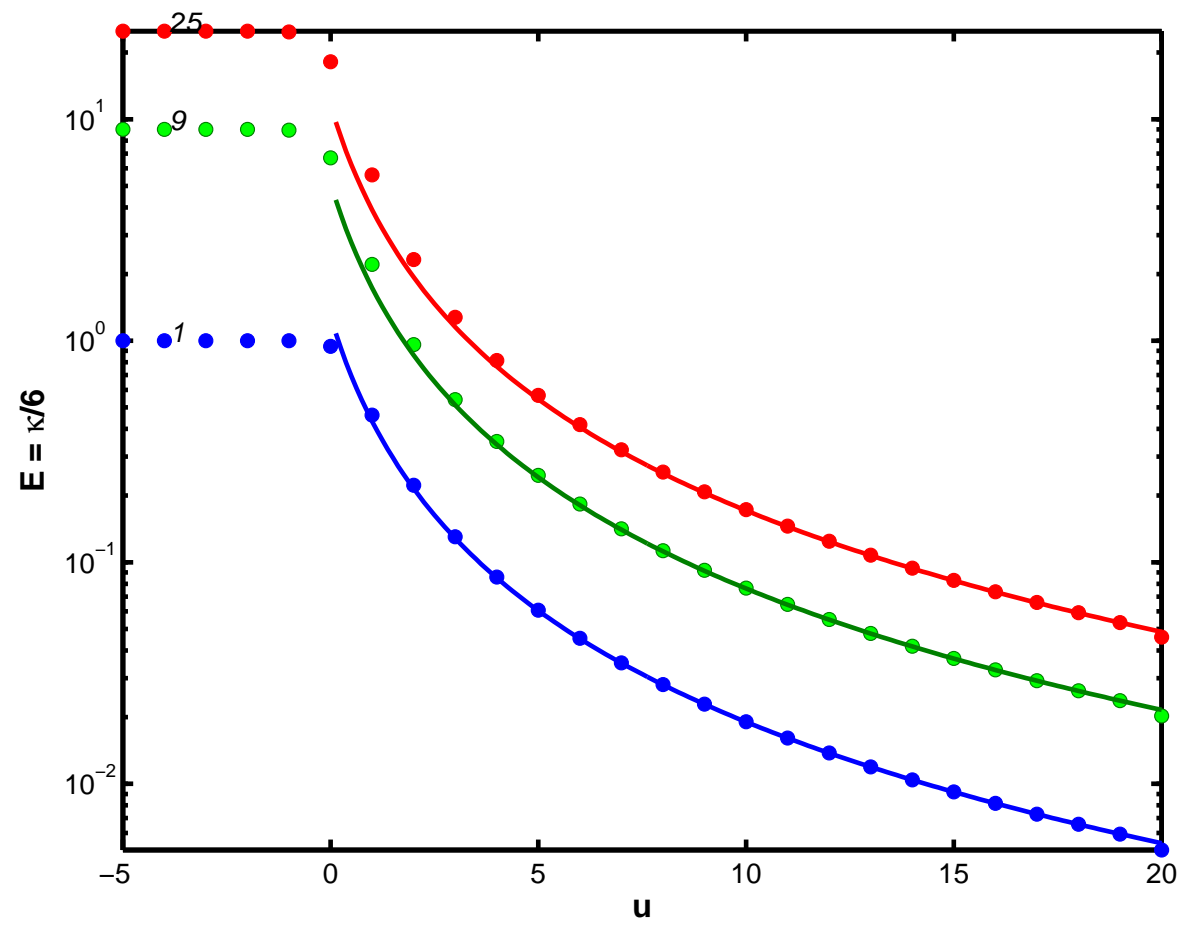

FIgURE 4. The asymptotic behavior in the $U V$ for the low lying states at $m=0$ : lines $\rightarrow E q$. (46), circles from numerical solution Eq. (55)).

the effective central charge of the SM (12) admits the exact (i.e. to all loops) calculation using the TBA equations. These equations can be derived from the factorized scattering theory of right $(r)$ and left $(l)$ moving massless particles which form the spectrum of our 


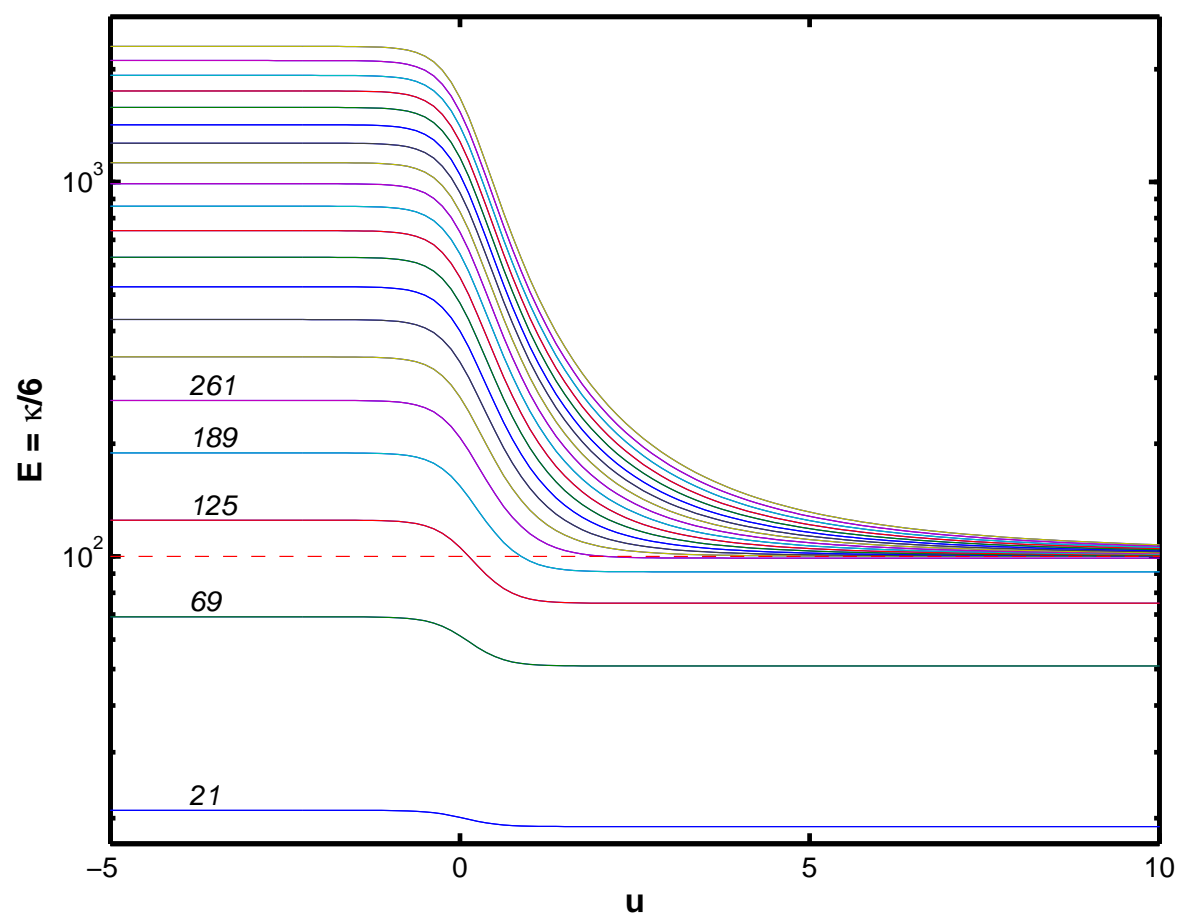

FiguRE 5. The spectrum flow for $m=10$; dashed line shows the continuum threshold.

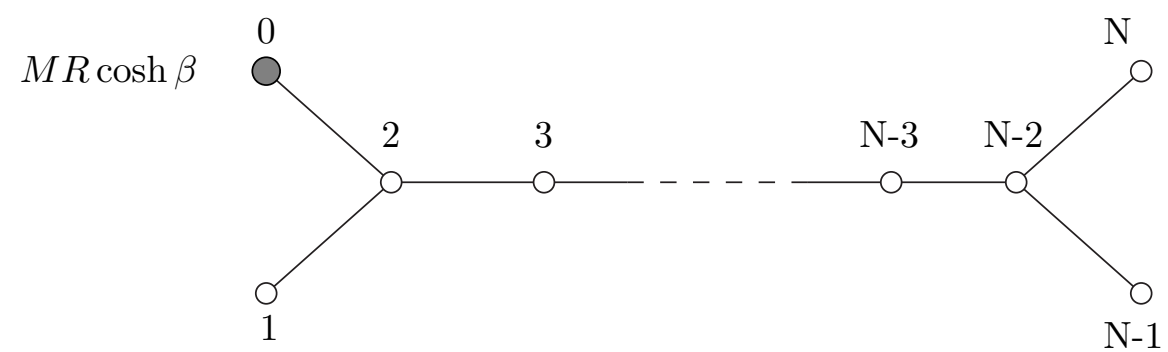

Figure 6. The extended Dynkin diagram for the Sausage model.

SM. The factorized scattering theory of massless particles is characterized by two-particle $S$-matrices $S_{r r}(\beta), S_{l l}(\beta)$, and $S_{r l}(\beta)$ where $\beta$ is the relative rapidity of scattering particles. We suppose to discuss in more details this scattering theory (as well as perturbed CFT approach to our SM) in another publication. Here we only note that each of these three $S$ matrices coincides formally with the $S$-matrix for the massive particles in $Z_{N}$ parafermionic CFT perturbed by the parafermionic operators. This $S$-matrix is described in details in Refs. 29, 30]. This scattering theory results in TBA equations which form the common system of $N+1$ coupled non-linear integral equations for $N+1$ functions $\varepsilon_{a}(\beta)$ of rapidity variable $-\infty<\beta<\infty$. The TBA system has the form:

$$
\rho_{a}(\beta)=\varepsilon_{a}+\frac{1}{2 \pi} \int \sum_{b=0}^{N} \varphi_{a b}\left(\beta-\beta^{\prime}\right) \log \left(1+e^{-\varepsilon_{a}\left(\beta^{\prime}\right)}\right) \mathrm{d} \beta^{\prime},
$$




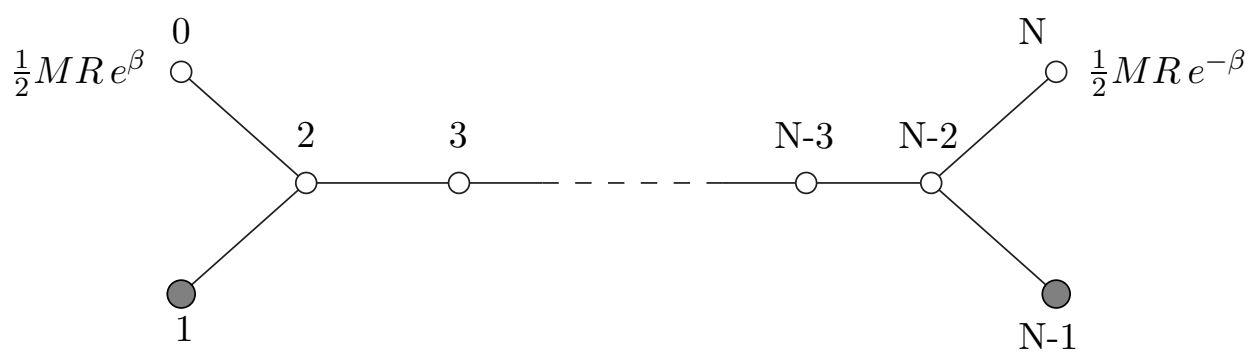

Figure 7. The extended Dynkin diagram for the SM(12)

where $\varphi_{a b}(\beta)=\frac{1}{2 \pi} \mathcal{I}_{a b} / \cosh \beta, \mathcal{I}$ being the incidence matrix of the extended affine $D_{N}$ Dynkin diagram (see Fig. 7) and the source term

$$
\rho_{a}(\beta)=\frac{1}{2} R M \exp (\beta) \delta_{a 0}+\frac{1}{2} R M \exp (-\beta) \delta_{a N} .
$$

The effective central charge can be calculated as

$$
c(R)=\frac{3}{\pi^{2}} \int \sum_{a} \rho_{a}(\beta) \log \left(1+\exp \left(-\varepsilon_{a}(\beta)\right) \mathrm{d} \beta .\right.
$$

The incidence matrix of these TBA equations is similar to that for the sausage model (see Fig. 6), the only difference coming from the source terms. For the massive sausage model (without topological term) $\rho_{a}(\beta)=R M \cosh (\beta) \delta_{a 0}$.

The UV behavior of $c(R)$ is determined only by the structure of the incidence matrix and we can find that in both cases effective central charge approaches to the limiting value $c_{U V}=c(0)=2$ logarithmically (see Ref.[11]) in agreement with Eqs. (4647). The analysis of TBA equations with source $\rho_{a}$ given by Eq. (61) shows that in the IR limit $c_{I R}=c(\infty)=$ $2-6 /(N+2)$ coincides with central charge of parafermionic CFT and the IR corrections to this value have a structure:

$$
\begin{aligned}
c(R, N)= & 2-\frac{6}{N+2}+b_{2}(N)\left(\frac{N+2}{M R}\right)^{8 /(N+2)}+ \\
& b_{3}(N)\left(\frac{N+2}{M R}\right)^{12 /(N+2)}+\ldots
\end{aligned}
$$

The IR asymptotics of our theory can be described by the methods of perturbed CFT. This field theory is characterized by integrable perturbative operator which belongs to the space of fields of the parafermionic CFT and has the dimension $\Delta_{\text {pert }}=1+2 /(N+2)$. It gives us the possibility to calculate analytically the first corrections to the expansion (63). The exact values for the coefficients $b_{2}(N)$ and $b_{3}(N)$ are presented in Appendix C.

The effective central charge $c(R, N)$ was computed using the TBA equations numerically for several values of $N(N=5,7,11,15,23, \ldots)$. At large $N$ the central charge is predicted to be given by

$$
c(R, N)=2-\frac{\kappa_{0}(u)}{N+2}+O(1 / N \log N)
$$

To verify this we have to relate $u$ to the parameter $M R$ entering the TBA equations. Within one loop approximation we have a freedom which can be used to fit the data in the best way. Asymptotically we expect $u \sim \log (N / M R) / N$, but finite $N$ corrections are present 
and may be important. Empirically we find that a rather accurate choice is the following

$$
\begin{aligned}
u & =N_{\text {eff }}^{-1} \log (N / M R) \\
N_{\text {eff }} & =\sqrt{(N+2)(N-2 \tanh (4 \log (N / M R)))}
\end{aligned}
$$

which is used to build the plot of Fig. 9, It is quite evident that the data are increasingly well matched by $\kappa$ as $N$ increases (in the deep UV or IR the agreement is even better).

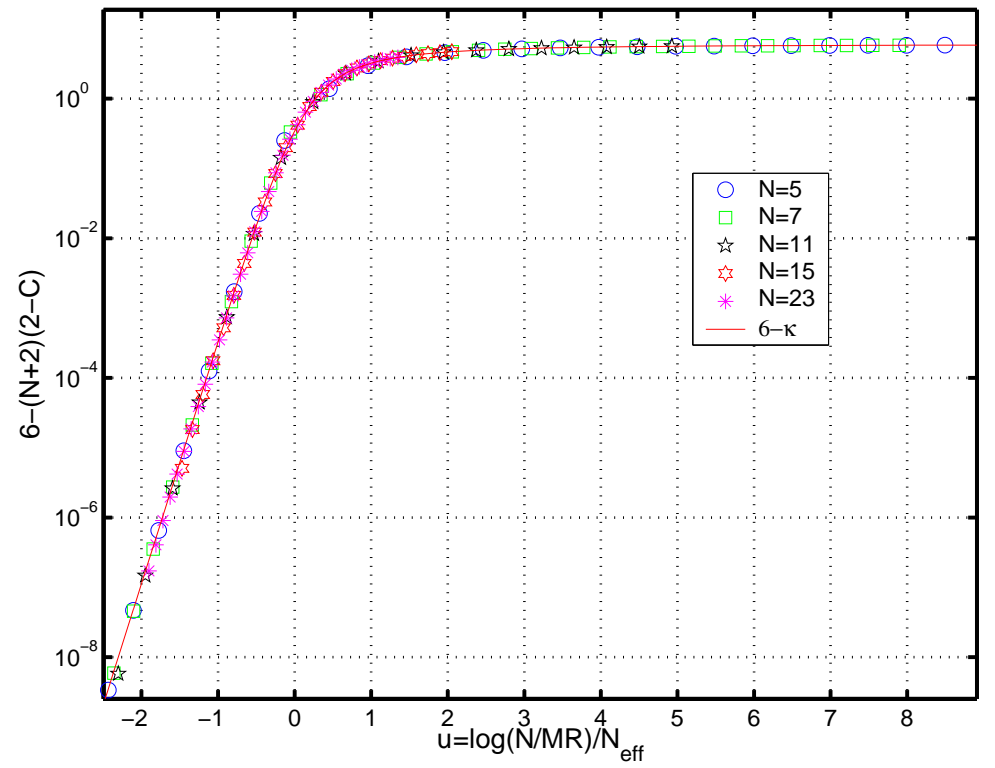

Figure 8. Matching $\kappa$ to the TBA data.

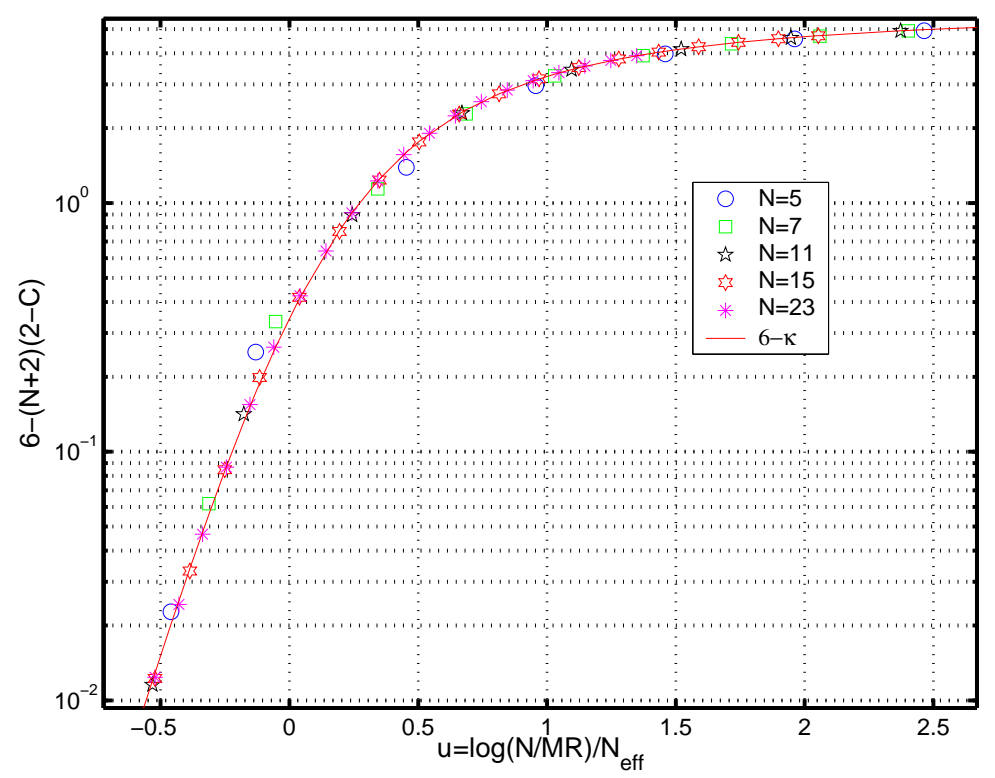

Figure 9. A close-up view of Fig.9. 
Finally let us note that the exact values of the coefficients $b_{2}(N)$ and $b_{3}(N)$, given in Appendix $\mathrm{C}$, have been reproduced with a high degree of accuracy by fitting the TBA data at various values of $N$.

\section{Conclusions}

We have found a unified treatment of the general spectral problem for the sausage model and its variant SM (12). The two formulations correspond to the same Heun equation defined on different domains in complex plane and they are linked by a projective transformation. The spectral function $\kappa(u)$ can be studied both numerically by diagonalizing a discretized form of the differential operator, or analytically by a power series expansion around the IR point. The perturbative expansion can be pushed to high orders and it turns out to be convergent in the whole physical domain. Its asymptotic behavior at high order is compatible with the leading UV behavior, which has been computed analytically. In the ultraviolet regime, the spectrum, besides the expected continuous component, contains a set of bound states with angular momentum higher than one. The scaling functions for the ground state has been compared to the central charge computed via TBA equations: a very good agreement was found, adding good evidence for the interpretation of the SM (12) (at $\nu=4 \pi / N)$ as the field theory describing the RG flow to the $Z_{N}$ parafermionic CFT.

\section{ACKNOWLEDGMENTS}

E.O. would like to thank R. K. Ellis and his colleagues of the Theory Group at Fermilab for the kind hospitality he enjoyed while this work was done. We warmly thank R. De Pietri who developed the symbolic code. This work was supported in part by the EU under contract HPRN-CT-2002-00325 and grant INTAS-OPEN-00-00055. 


\section{APPENDiX A}

We recall some standard results from Raileigh-Schroedinger perturbation theory for nondegenerate levels, which should be found in any textbook on Quantum Mechanics. Stationary perturbation theory is most efficiently formulated as an iterative algorithm. Let $H=H_{0}+\varepsilon V, E_{0}$ any one of the unperturbed eigenvalues, with eigenstate $\left|E_{0}\right\rangle$. Then the perturbed eigenvalue $E(\varepsilon)$ can be expanded as

$$
E(\varepsilon)=E_{0}+\sum_{n \geq 1} \delta_{n} \varepsilon^{n}
$$

while the eigenstate is given by a vector series in terms of auxiliary vectors $\left|\eta_{n}\right\rangle$ as

$$
|E(\varepsilon)\rangle=\left|E_{0}\right\rangle+\sum_{n \geq 1} \varepsilon^{n}\left|\eta_{n}\right\rangle
$$

The expansion coefficients can be computed through the following recursive algorithm:

Let $\left|\eta_{0}\right\rangle=\left|E_{0}\right\rangle$ and $\mathcal{R}_{0}=\frac{1-\left|E_{0}\right\rangle\left\langle E_{0}\right|}{H_{0}-E_{0}} ;$ then for any $n \geq 1$ we have

$$
\left\{\begin{array}{l}
\delta_{n}=\left\langle E_{0}|V| \eta_{n-1}\right\rangle \\
\left|\eta_{n}\right\rangle=\mathcal{R}_{0}\left\{-V\left|\eta_{n-1}\right\rangle+\sum_{k=1}^{n} \delta_{k}\left|\eta_{n-k}\right\rangle\right\}
\end{array}\right.
$$

The algorithm can be easily translated in any symbolic manipulation language. In principle we should take care of defining the matrix $V$ in an orthonormal basis; this would introduce some square roots in our matrix Eq. (22), while at the end the coefficients turn out to be rational. Actually the following lemma tells us that we may comfortably work with the unnormalized basis.

Lemma: Let $H, H_{0}, V,\left|E_{0}\right\rangle, E(\varepsilon), \mathcal{R}, \delta_{n},\left|\eta_{n}\right\rangle$ be defined as above. Let us assume further, to avoid any convergence problem, that $V$ be a finite-band matrix along the main diagonal. Let $S$ be any nonsingular Hermitian operator commuting with $\mathcal{H}_{0}$. Then we may substitute $V$ with $S V S^{-1}$ in the iterative algorithm (69), leaving everything else unchanged.

Proof: Let $\delta_{n}^{\prime},\left|\eta_{n}^{\prime}\right\rangle$ denote the sequence constructed by inserting $S V S^{-1}$ into eq. (69). Since $S$ commutes with $H_{0}$, we must have $S\left|E_{0}\right\rangle=s_{0}\left|E_{0}\right\rangle$ for some non-zero $s_{0}$. Hence

$$
\delta_{n}^{\prime}=\left\langle E_{0}\left|V s_{0} S^{-1}\right| \eta_{n-1}^{\prime}\right\rangle .
$$

Let us define $\left|\eta_{n}^{\prime \prime}\right\rangle=s_{0} S^{-1}\left|\eta_{n-1}^{\prime}\right\rangle$. It's easy to check that the sequence $\left\{\delta_{n}^{\prime},\left|\eta_{n}^{\prime \prime}\right\rangle\right\}$ satisfies the same recursion as $\left\{\delta_{n},\left|\eta_{n}\right\rangle\right\}$, and moreover $\left|\eta_{0}^{\prime \prime}\right\rangle=s_{0} S^{-1}\left|E_{0}\right\rangle=\left|E_{0}\right\rangle$, hence the two sequences must coincide.

Note: the statement in the lemma is strictly perturbative. $H$ and $S H S^{-1}$ could be inequivalent as operators, since $S$ and/or $S^{-1}$ may be unbounded, still they share the same perturbative expansion.

\section{APPENDix B}

We report the explicit expression of the series expansion of $\kappa_{m, n}$ for small values of $(n, m)$. Of course this is just a sample; the code can generate them to any order, the only limitation 
being computer's physical memory and time.

$$
\begin{array}{rl}
\kappa_{0,0}= & 6-\lambda^{2}-\frac{1}{2} \lambda^{3}-\frac{229}{720} \lambda^{4}-\frac{109}{480} \lambda^{5}-\frac{62999}{362880} \lambda^{6}-\frac{20159}{145152} \lambda^{7}-\frac{299803787}{2612736000} \lambda^{8} \\
& -\frac{72503387}{746496000} \lambda^{9}-\frac{173336436487}{2069286912000} \lambda^{10}+O\left(\lambda^{11}\right) \\
\kappa_{1,0}= & 18-4 \lambda-\frac{16}{9} \lambda^{2}-\frac{352}{405} \lambda^{3}-\frac{1972}{3645} \lambda^{4}-\frac{17408}{45927} \lambda^{5}-\frac{701314}{2460375} \lambda^{6} \\
& -\frac{34835788}{155003625} \lambda^{7}-\frac{204567413}{1116026100} \lambda^{8}-\frac{1588445666493}{10358117240625} \lambda^{9} \\
& -\frac{4782354354298021}{36543437624925000} \lambda^{10}+O\left(\lambda^{11}\right) \\
\kappa_{0,1}= & 54-24 \lambda-\frac{27}{5} \lambda^{2}-\frac{27}{10} \lambda^{3}-\frac{23949}{14000} \lambda^{4}-\frac{34047}{28000} \lambda^{5}-\frac{370287}{400000} \lambda^{6} \\
& -\frac{826209}{1120000} \lambda^{7}-\frac{146655243891}{241472000000} \lambda^{8}-\frac{35351959491}{68992000000} \lambda^{9} \\
& -\frac{197594782006203}{448448000000000} \lambda^{10}+O\left(\lambda^{11}\right) \\
\kappa_{1,1} & 90-\frac{196}{5} \lambda-\frac{9664}{1125} \lambda^{2}-\frac{7627904}{1771875} \lambda^{3}-\frac{2173866688}{79734375} \lambda^{4}-\frac{173655964928}{8970171871875} \lambda^{5} \\
& -\frac{485256409132928}{329651806640625} \lambda^{6}-\frac{955858372577612032}{815888221435546875} \lambda^{7}-\frac{176847414696606187696}{183574849822998046875} \lambda^{8} \\
& -\frac{1281558049490242326541456}{15786519210528717041015625} \lambda^{9}+O\left(\lambda^{10}\right)
\end{array}
$$

\section{Appendix C}

In this appendix we give the exact values for the first IR correction to the levels $e_{m j}(R)$ and two first corrections to the effective central charge or to the ground state energy $e_{0}(R)$. We express these corrections in terms of parameter $M$ entering the TBA equations. These corrections can be calculated analytically using the methods of integrable perturbed CFT. We consider the case $\nu / 4 \pi=1 / N$. In this case the IR limit of SM (12) is described by parafermionic CFT. The conformal dimensions of the primary fields in this CFT are characterized by two quantum numbers $m$ and $j(j=|m / 2|,|m / 2|+1 \ldots \leq N / 2)$ and have a form:

$$
\Delta_{m j}=\frac{j(j+1)}{N+2}-\frac{m^{2}}{4 N}
$$

It is convenient to introduce $D_{m j}=(N+2) \Delta_{m j}$. As it was noticed in the Introduction, the limiting values of $\left(e_{m j}-e_{0}\right) / 24$ should coincide with $\Delta_{m j}$. With the first IR correction these values are:

$$
\frac{(N+2)\left(e_{m j}(R)-e_{0}(R)\right)}{6}=4 D_{m j}-D_{m j}^{2} \frac{b_{1}(j, N)}{j(j+1)}\left(\frac{N+2}{M R}\right)^{4 /(N+2)}+\ldots
$$

where the coefficient $b_{1}(j, N)$ can be expressed through the function $g(x)=\Gamma(1+x) / \Gamma(1-x)$ and has a form

$$
b_{1}(j, N)=\frac{2 N^{2}}{(N+2)^{2}} \frac{g\left(\frac{1}{N+2}\right)^{2} g\left(\frac{2 j+2}{N+2}\right)}{g\left(\frac{2}{N+2}\right) g\left(\frac{2 j}{N+2}\right)}(8 \pi)^{4 /(N+2)} .
$$

We note that in the large $N$ limit the left hand side of Eq.(172) tends to $\left(\kappa_{m j}-\kappa_{0}\right) / 6$. For $4 D_{m j}$ and $b_{1}(j, N)$ we have

$$
4 D_{m j}=\left(4 j(j+1)-m^{2}\right)(1+O(1 / N)), \quad b_{1}(j, N)=2+O(1 / N) ;
$$

if we now define the parameter $u$ by the relation: $\left(\frac{N+2}{M R}\right)^{4 /(N+2)}=\exp (4 u)=\lambda /(1-\lambda)$ we can see that the first term in the expansion given in Eq. (33) coincides with Eq.(72) at one loop accuracy. 
For the effective central charge $c(R, N)$ (or for ground state level $e_{0}(R)$ ) it is possible to calculate analytically two further IR corrections. Namely, the coefficients $b_{2}$ and $b_{3}$ in the expansion (63) are

$$
\begin{aligned}
(N+2) b_{2}(N) & =\frac{N^{2}(N-2)^{2} g\left(\frac{1}{N+2}\right) g\left(\frac{3}{N+2}\right)(8 \pi)^{8 /(N+2)}}{(N+4)^{2}(N+6)^{2} g\left(\frac{4}{N+2}\right) g\left(\frac{-2}{N+2}\right)^{2}}=1+O(1 / N) ; \\
(N+2) b_{3}(N) & =-\frac{3 N^{4}(N-4)^{2} g\left(\frac{2}{N+2}\right) g\left(\frac{4}{N+2}\right)(8 \pi)^{12 /(N+2)}}{2(N+4)^{4}(N+8)^{2} g\left(\frac{6}{N+2}\right) g\left(\frac{-3}{N+2}\right)^{2}}=-\frac{3}{2}+O(1 / N) .
\end{aligned}
$$

It is easy to see from these equations that with one loop accuracy the IR expansion for the function $\kappa_{0}(u)=6-\lambda^{2}-\lambda^{3} / 2+\ldots$ coincides with exact IR expansion for the function $(N+2)(2-c(R, N))$.

In the UV limit the leading term for the levels $e_{m j}(R)$ (for $j \geq m-1 / 2$ ) can also be calculated exactly and has a form:

$$
N e_{m j}(R)=6 m^{2}+\frac{3(j-m+1)^{2} \pi^{2} N(N-2)}{2 Z_{m}^{2}(R)}+O\left(1 / Z_{m}^{5}\right)
$$

where $Z_{m}(R)=\log (8 \pi(N-2) / R M)+(N-2)\left(\psi(1)-\psi\left(\frac{m+1}{2}\right)\right)+\psi(1)$. This asymptotic behavior coincides at one loop accuracy with Eq.(46), where $n=j-m / 2$.

\section{REFERENCES}

[1] A.M. Polyakov, Phys. Lett. B59 (1975) 79.

[2] E. Brezin and J. Zinn-Justin, Phys. Rev. B14 (1976) 3110.

[3] D. Friedan, Phys. Rev. Lett. 45 (1980) 691.

[4] J. Zinn-Justin, "Quantum field theory and critical phenomena" (Oxford Science Publication, Oxford 1989).

[5] E. Fradkin and A. Tseitlin, Ann. Phys, 143 (1982) 413.

[6] C. Callan, D. Friedan, E. Martinec and M. Perry, Nucl. Phys. B262 (1985) 593.

[7] C. Lovelace, Phys. Lett. B135 (1984) 75.

[8] P. Candelas, G. Horowitz, A. Strominger and E. Witten, Nucl. Phys. B261 (1985) 46.

[9] C.N. Yang and C.P. Yang, J. Math. Phys. 10 (1969) 1115.

[10] Al. B. Zamolodchikov, Nucl. Phys. B342 (1990) 695.

[11] V. A. Fateev, E. Onofri and Al. B. Zamolodchikov, Nucl. Phys. B 406, 521-565 (1993).

[12] A. B. Zamolodchikov, JETP Lett. 43 (1986) 565.

[13] G. Perelman, The entropy formula for Ricci flow and geometric applications, arXiv:math.DG/0211159.

[14] E. Kiritis, Mod. Phys. Lett., A6 (1991) 2871.

[15] J. Maldacena, G. Moore and N. Seiberg, JHEP, 2001:0107, 46 (hep-th/0108044).

[16] V. Fateev and A. B. Zamolodchikov, Sov. Phys. JETP. 63 (1986) 913.

[17] R. Brunelli and G. P. Tecchiolli, J. of Comp. and Appl. Mathematics 57 (1995) 329-343.

[18] K. Heun, Math.Ann. 33 (1888) 161-179.

[19] E. L. Ince, "Ordinary Differential Equations", Dover, New York, (1956).

[20] A. Erdélyi, Ed., "Higher Trascendental Functions", McGraw-Hill, New York, 1955.

[21] G. H. Golub and C. F. Van Loan, "Matrix computations", 3rd Ed.n, Johns Hopkins Univ. Press (1996).

[22] Y. L. Luke, "The Special Functions and their approximations", Academic Press, New York, 1969, Vol.1.

[23] E. Kahmke, "Differentialgleichungen", Band 1, Chelsea Pub. Co., N.Y. 1959.

[24] T. Kato, "Perturbation Theory for Linear Operators", Springer-Verlag, Berlin 1995.

[25] M. Reed and B. Simon, "Methods of Modern Mathematical Physics", Vol.IV, Academic Press, New York, 1978.

[26] G. H. Hardy, "Divergent Series", Oxford U.P., 1949.

[27] R. Dijkgraaf, H. Verlinde and E. Verlinde, Nucl. Phys. B 371 (1992) 269.

[28] E. Witten, Phys. Rev. D 44 (1991) 314.

[29] V. A. Fateev, Int. J. Mod. Phys. A16 (1991) 2109.

[30] V. A. Fateev and Al. B. Zamolodchikov, Phys. Lett. B271 (1991) 91. 